\title{
Diffusion weighted imaging in prostate cancer
}

\author{
Cher Heng Tan • Jihong Wang • Vikas Kundra
}

Received: 21 April 2010 /Revised: 5 July 2010 /Accepted: 6 August 2010/Published online: 9 October 2010

(C) European Society of Radiology 2010

\begin{abstract}
Diffusion-weighted imaging has generated substantial interest in the hope that it can be developed into a robust technique to improve the accuracy of MRI for the evaluation of prostate cancer. This technique has the advantages of short acquisition times, no need for intravenous administration of contrast medium, and the ability to study diffusion of water molecules that indirectly reflects tissue cellularity. In this article, we review the existing literature on the utility of DWI in tumour detection, localisation, treatment response, limitations of the technique, how it compares with other imaging techniques, technical considerations and future directions.
\end{abstract}

C. H. Tan · V. Kundra

Department of Diagnostic Radiology, Division of Diagnostic Imaging, The University of Texas, M D Anderson Cancer Center, 1515 Holcombe Blvd,

Houston 77030 TX, USA

\section{J. Wang}

Department of Imaging Physics, Division of Diagnostic Imaging, The University of Texas, M D Anderson Cancer Center,

1515 Holcombe Blvd,

Houston 77030 TX, USA

V. Kundra

Department of Experimental Diagnostic Imaging,

Division of Diagnostic Imaging, The University of Texas,

M D Anderson Cancer Center,

1515 Holcombe Blvd,

Houston 77030 TX, USA

email: vkundra@mdanderson.org

Present Address:

C. H. Tan $(\bowtie)$

Department of Diagnostic Radiology, Tan Tock Seng Hospital,

11 Jalan Tan Tock Seng,

Singapore 308433, Singapore

e-mail: tchers1977@gmail.com
Keywords Magnetic resonance imaging $\cdot$ Functional Diffusion $\cdot$ Prostate $\cdot$ Cancer

\section{Background}

Prostate cancer (PC) is the most common cancer among adult males. In the United States, it is estimated that 192,280 men will be diagnosed with and 27,360 men will die of PC in 2009 [1]. At present, serum prostate-specific antigen (PSA) levels and digital rectal examination (DRE) are the mainstays of clinical detection of PC. Abnormal findings are followed up by transrectal ultrasound (TRUS)guided biopsy. Therapy generally consists of local therapy such as surgery or radiation therapy for localised disease and predominantly hormonal therapy for metastatic disease followed by taxane-based chemotherapy when hormonal therapy fails.

\section{Clinical application of MRI in localised prostate cancer}

Magnetic resonance imaging (MRI) is more accurate than either DRE or TRUS biopsy in preoperative anatomical localisation of PC [2]. MRI provides incremental value to clinical findings in staging patients of intermediate risk. For example, organ-confined disease implies that the patient may benefit from local therapy such as surgery [3]. In cases where there is discordance between PSA levels and biopsy findings (e.g. elevated PSA levels but negative biopsy), MRI may be used to detect and direct re-biopsy of suspected tumour foci [4]. When tumour recurrence is suspected, MRI can aid in localising the site of tumour recurrence [5]. 


\section{Conventional MRI for localised prostate cancer evaluation}

High-resolution fast spin-echo MRI using an endorectal coil provides excellent visualisation of the prostate gland and surrounding structures. Tumour appears hypointense to normal prostate tissue on T2-weighted images (T2WI). Prostatitis, benign prostatic hyperplasia (BPH) and posttreatment changes (radiation and hormone ablative therapy) are common false-positive findings [6]. The sensitivity and specificity of T2WI for PC vary widely, due to differences in imaging techniques, reference standards, criteria for defining disease involvement on MRI and inter-observer variability [7]. In a meta-analysis by Sonnad et al., T2WI showed a maximum joint sensitivity and specificity rate of $74 \%$ for the staging of PC [8]. Engelbrecht et al. [7] showed similar findings, with a joint sensitivity and specificity rate of $71 \%$.

Clearly, T2WI has limitations in PC evaluation. This has led to substantial interest in advanced MRI techniques such as diffusion-weighted imaging (DWI), magnetic resonance spectroscopy (MRS), and dynamic contrast-enhanced (DCE)-MRI as adjuncts to T2WI.

\section{DWI methodology for prostate MRI}

For this paper, relevant English language publications in the Medline database pertaining to DWI of the prostate gland, up to as recent as March 2010, were carefully reviewed.

DWI is potentially useful in tumour detection, staging, assessment of treatment response and disease relapse [9]. It measures the restriction of diffusion in biological tissues and properties such as cellular density, membrane permeability, and space between cells; it can aid in distinguishing benign from malignant tissues. For example, the luminal space in benign human prostate tissue is on average hundreds of microns wide, while the restrictions to water diffusion in PC is up to ten microns, a displacement scale at which DWI is sensitive [10].

Because the prostate gland is not subject to much motion (e.g. cardiac, respiratory or bowel), MRI, including DWI, can be performed without breath-holding. Typically, an ultrafast echoplanar T2-weighted sequence is used (Table 1). Either endorectal or phased-array coils can be employed.

Diffusion-weighted images can be post-processed to obtain apparent diffusion coefficients (ADCs). ADCs eliminate the effect of $\mathrm{T} 2$ shine-through, and objectively measures tissue diffusivity [11]. ADC maps allow for visual assessment; the tumour appears as an area of decreased signal (Fig. 1).

Diffusion-weighted imaging has the distinct advantage of being acquired very rapidly, without the use of intravenous contrast material or specialised hardware. In a recent study, using $1.5 \mathrm{~T}$ and an endorectal coil for imaging, total image acquisition time was less than $1.5 \mathrm{~min}$ for imaging at 4-mm sections (12 slices) across the entire gland, at multiple b-values of $0,300,500$ and $800 \mathrm{sec} / \mathrm{mm}^{2}$ [12].

The advent of parallel imaging coils and advancements in echo-planar imaging in recent years have allowed for further shortening of echo-train lengths (and consequently imaging times) and improved spatial resolution [13]. At a $256 \times 256$ matrix and slice thickness of $3 \mathrm{~mm}$ without an intersection gap (performed at $1.5 \mathrm{~T}$ using an endorectal coil), spatial resolutions of $10.8 \mathrm{~mm}^{3}$ are achieved [14].

\section{Potential uses of DWI in prostate cancer detection}

Lesions within the peripheral zone

Most of the studies to date that have assessed the utility of DWI in PC evaluated lesions within the peripheral zone (PZ). Several have suggested improvement in tumour detection over T2WI, either using DWI alone, or in combination with T2WI. Mazaheri et al. demonstrated high sensitivity $(82 \%)$ and specificity rates $(95 \%)$ using an ADC cut-off of $1.6 \times 10^{-3} \mathrm{~mm}^{2} / \mathrm{s}$ [15], while improving tumour volume estimation. Miao et al. [16] showed that the sensitivity and specificity rates for T2WI was $76 \%$ and $70 \%$ respectively, compared with $86 \%$ and $80 \%$ for DWI. Combined T2WI and DWI yield slightly better results, with reported sensitivity and specificity rates of $45-89 \%$ and $61-97 \%$ respectively, compared with $74-85 \%$ and $57-95 \%$ respectively for DWI alone and $25-87 \%$ and $57-92 \%$ respectively for T2WI alone [12, 15, 17-26].

Lesions within the transitional zone

On T2WI, PZ is distinctly separate from the transitional zone (TZ), which would theoretically include the anatomic central zone as well. It is accepted that tumour detection by T2WI in the transitional zone (TZ) is inferior to that in the PZ [27]. The $\mathrm{PZ}$ is predominantly composed of glandular tissue that is hyperintense on T2WI and contrasts well with tumour. In comparison, the $\mathrm{TZ}$ contains more stromal tissue, giving rise to lower T2 signal which may overlap with tumour [28].

Diffusion-weighted imaging may improve MRI detection of TZ tumours (Fig. 2). ADC values for malignant tissue are commonly lower than those of normal PZ and TZ [27, 29], as well as BPH nodules [30]. Shimofusa et al. [21] found that DWI detected tumour in the TZ in 5 out of $8(63 \%)$ patients, compared with one out of $8(13 \%)$ patients detected using T2WI. Yoshimitsu et al. found that the mean tumour volume in the TZ detected by DWI increased from $20 \%$ to $52 \%$ compared with T2WI $(p<0.0001)$ [17]. 
Table 1 Example of imaging parameters using an endorectal coil at $1.5 \mathrm{~T}$

\begin{tabular}{ll}
\hline Parameter & Value \\
\hline Field of view (cm) & $14 \times 14$ \\
Matrix size & $128 \times 128$ \\
TR (msec) & 8000 \\
TE (msec) & $\sim 70-80$ \\
Bandwidth & $167 \mathrm{~Hz}$ \\
Number of signals acquired & 3 \\
Section thickness (mm) & 5 \\
Interslice gap (mm) & 0 \\
Direction of motion probing & Phase, frequency, and section \\
gradients & $(3 \mathrm{scan}$ trace) \\
b-values (sec/mm ${ }^{2}$ ) & 0,700 \\
Acquisition time (minutes) & $\sim 3.5$ \\
\hline
\end{tabular}

However, as with T2WI, DWI sensitivity for tumour in the TZ remains less than in the PZ. In a study of 47 patients, Kim and colleagues [25] found that the mean ADC value for normal TZ $\left(1.441 \times 10^{-3} \mathrm{~mm}^{2} / \mathrm{s}\right)$ was significantly higher than for the PZ $\left(1.146 \times 10^{-3} \mathrm{~mm}^{2} / \mathrm{s}\right)$. In that study, sensitivity of ADC values for PC in the peripheral zone was $98 \%$, compared with $81 \%$ in the transitional zone $(p=0.01)$, although there were no significant differences in specificity.

\section{Extraprostatic involvement}

Seminal vesicle invasion (SVI) (Fig. 3) is generally regarded as a poor prognostic indicator after radical prostatectomy [31]. Ren and colleagues [23] showed that the combined use of DWI with T2WI is superior to T2WI alone for detecting SVI, while noting that ADC values are lower in PC than in normal seminal vesicles. Kim et al. showed increased specificity (87\% to $97 \%$ ) and accuracy (87\% to $96 \%$ ) for SVI by combining DWI and T2WI compared with T2WI alone; substantial inter-reader agreement was found despite the presence of haemorrhage within the SV in a number of patients [18]. Interestingly, the increase in accuracy and specificity was greater for the less experienced reader.

In a similar study looking at urinary bladder involvement, PC had a significantly lower ADC value $\left(0.963 \times 10^{-3} \mathrm{~mm}^{2} / \mathrm{s}\right)$ than normal urinary bladder wall $\left(1.517 \times 10^{-3} \mathrm{~mm}^{2} / \mathrm{s}\right)$ and combining DWI with T2WI increased the sensitivity and specificity for urinary bladder invasion by PC (82\% and 78\%) compared with either T2WI alone (73\% and $65 \%$ ) or DWI alone (78\% and 57\%) [26].

\section{Pre-treatment detection: tumour localisation and prognostication}

Early detection and localisation of PC at a treatable stage is important. TRUS-guided biopsy in patients with elevated PSA levels has low sensitivity of around $60 \%$ and a high false-negative rate of $15-30 \%$ [32, 33]. DWI may be helpful in localising tumour in patients with previous failed TRUS biopsy, with the potential to improve rebiopsy success rates. In a multireader study of 17 patients with both previous negative TRUS biopsy and persistently elevated PSA before repeat biopsy, DWI at 3T combined with T2WI had improved sensitivity of $81-84 \%$ compared with 54-73\% for T2WI alone [20].

Tumour localisation should improve radiotherapy planning for escalating the dose to the tumour relative to the surrounding normal tissues. This has already been demonstrated for MRS [34, 35]. Kajihara and colleagues found that the addition of DWI to T2WI increased both sensitivity (from $26-51 \%$ to $47-72 \%$ ) and positive predictive value (from $47-73 \%$ to $58-80 \%$ ) in the localising $\mathrm{PC}$ and suggested that this may improve radiation therapy planning [19].
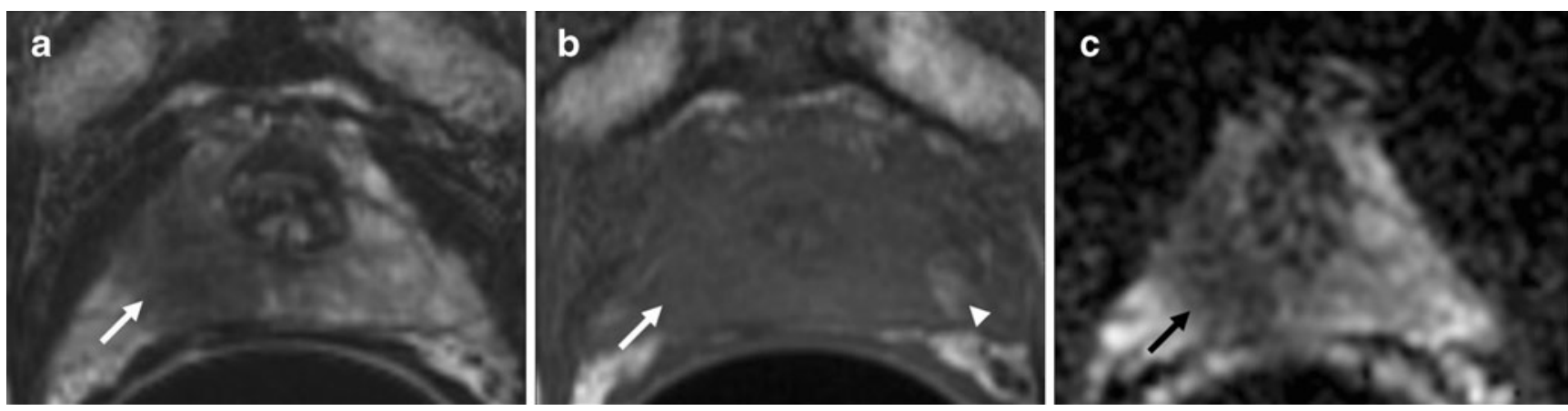

Fig. 1 Endorectal MRI prostate performed at $1.5 \mathrm{~T}$ in a 59-year-old man with prostate cancer. a T2WI at the level of the apex shows focal low signal in the peripheral zone on the right (arrow). b Corresponding T1WI shows isointense signal in the prostate, including on the right (arrow). A small amount of high signal is seen laterally on the left (arrowhead), consistent with haemorrhage. c ADC map shows a focal area of low signal in the apex on the right (arrow) 


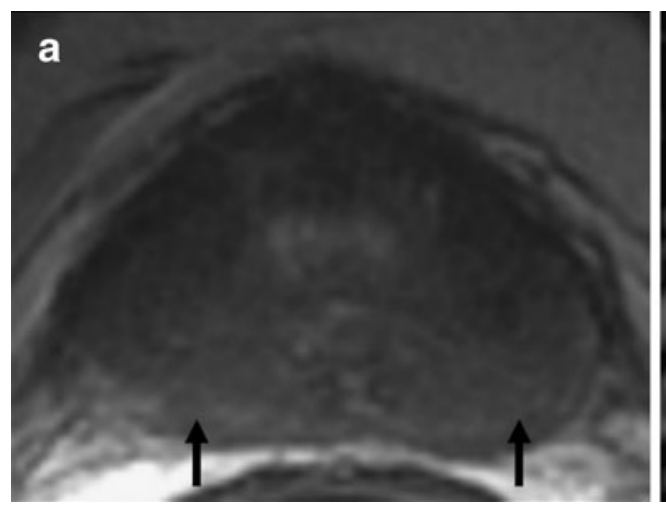

Fig. 2 Endorectal MRI of the prostate performed at 1.5T in a 52-yearold man with prostate cancer. a Axial T2WI of the prostate at the mid-gland level demonstrates diffuse hypointense signal involving the peripheral, which is inseparable from the transitional zone.

Detection of tumour in areas of haemorrhage following TRUS biopsy is a problem with T2WI. DWI (57\%) has been shown to be slightly more sensitive than T2WI (51\%) in detecting tumour within areas of haemorrhage [36]. Prostate cancer tends to have lower ADC signal than blood (Fig. 4).

Diffusion-weighted imaging may also play a role in PC prognosis. It has been shown that the ADC of poorlydifferentiated cancer $\left(0.93 \times 10^{-3} \mathrm{~mm}^{2} / \mathrm{s}\right)$ is lower than that of a well-differentiated cancer $\left(1.19 \times 10^{-3} \mathrm{~mm}^{2} / \mathrm{s}\right)$ [17]. Zelhof et al. [37] and Gibbs et al. [38] showed that ADC values correlate inversely with increasing cellularity within the prostate gland. DeSouza and colleagues [39] found that

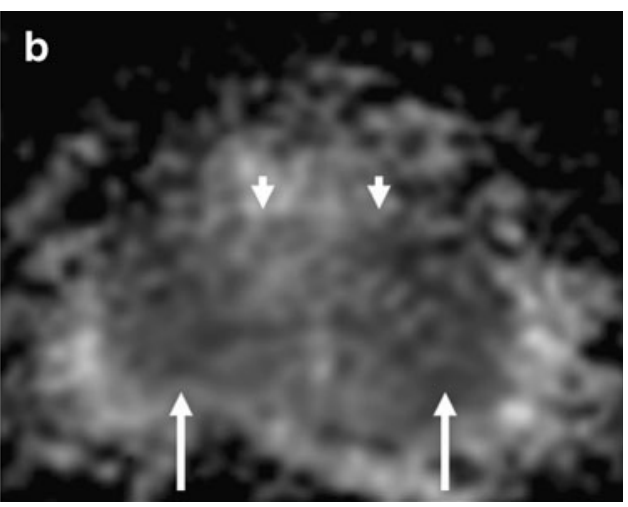

b Corresponding ADC map, demonstrates low signal in both the peripheral zone (long arrows) and the transitional zone (short arrows), is consistent with tumour infiltration in both locations

patients in higher risk groups with lower ADC values had a greater tumour burden.

\section{Post-treatment detection: monitoring treatment response and local tumour recurrence}

Local ablative therapies such as cryoablation, radiation therapy or high-intensity focused ultrasound (HIFU) can result in diffusely decreased $\mathrm{T} 2$ signal, potentially masking residual disease [40]. In experimental models, normal prostatic tissue that regenerated following thermal injury showed a rise in the ADC values towards normal [41].
Fig. 3 A 62-year-old man with seminal vesicle invasion by prostate cancer. MRI was performed using a $1.5 \mathrm{~T}$ scanner. a Axial T2WI of the seminal vesicles (arrow) showing normal high $\mathrm{T} 2$ signal.

b Corresponding ADC signal in the seminal vesicles (arrow) at the level of a. c Axial T2WI of seminal vesicles at a different level (arrow) showing diffuse loss of normal T2 signal consistent with seminal vesicle invasion by prostate cancer. d Corresponding ADC signal in the seminal vesicles (arrow) at the level of (c) demonstrates lower ADC signal compared with (b), consistent with seminal vesicle invasion by prostate cancer
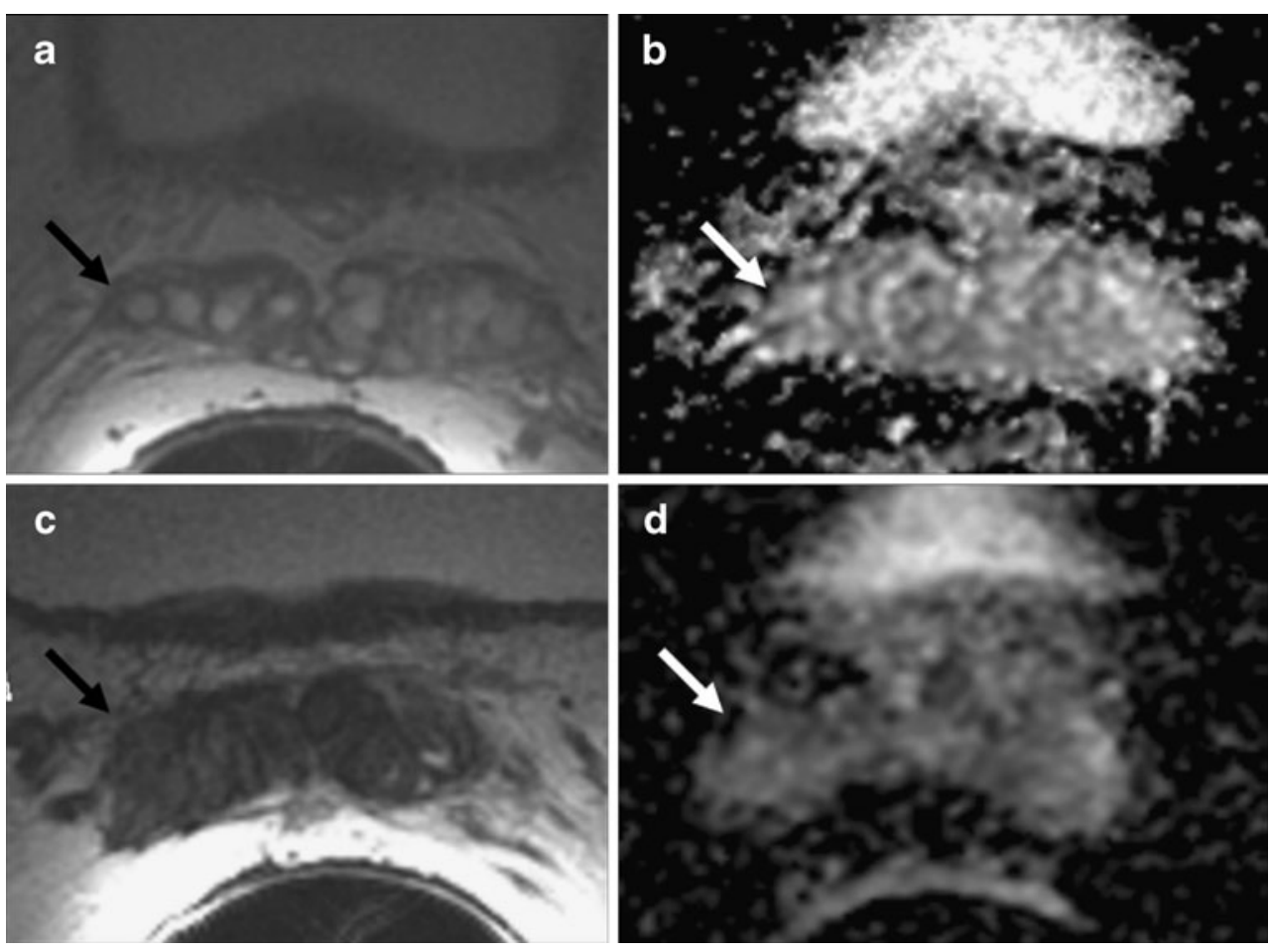
Fig. 4 A 74-year-old man with biopsy-proven prostate cancer and multifocal post-biopsy haemorrhage. MRI was performed using a $1.5 \mathrm{~T}$ scanner. a $\mathrm{T} 1 \mathrm{WI}$ shows focal hyperintense signal (arrow) in the right peripheral zone, consistent with haemorrhage. b Mild signal loss is present on the corresponding ADC map due to susceptibility artefact (arrow). c T2WI at a different level in the gland showing focal hypointense signal (arrow) consistent with a tumour. There was no evidence of increased signal at this site on T1WI (not shown). d ADC map of the gland at the same level as in (c) that is relatively lower in signal (arrow) in comparison to the haemorrhagic focus in (b)
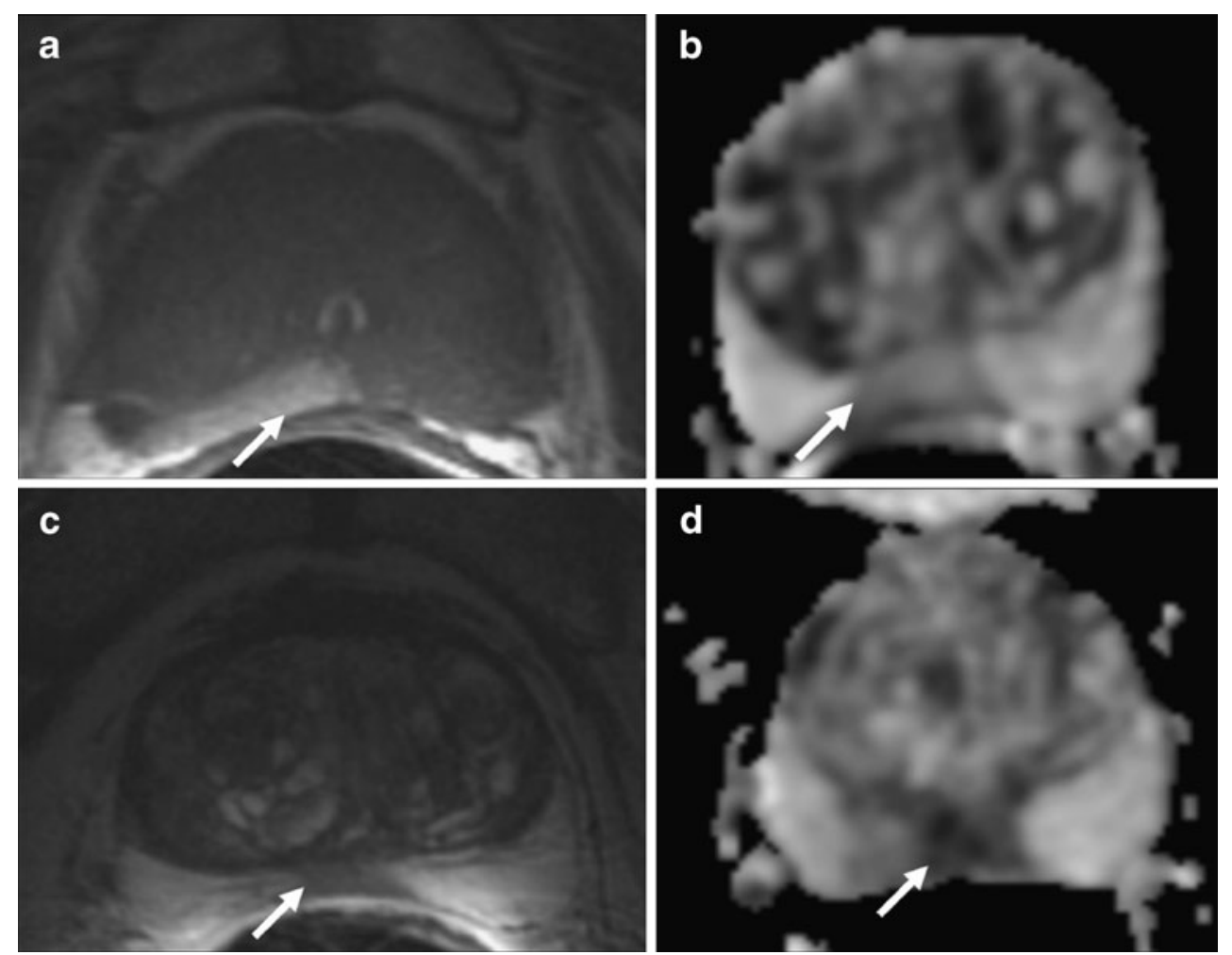

Therefore, sites of residual or recurrent disease that continue to show restricted diffusion can theoretically be detected by DWI.

In patients who underwent HIFU for PC, combined T2WI and DWI have been shown to be more specific than dynamic contrast-enhanced (DCE) MRI (74\%-78\% vs $63 \%-68 \%$ ) in detecting post-treatment tumour progression, even though sensitivity may be lower (63-70\% vs $80-87 \%$ ) [42]. Following external beam radiation therapy, DWI had higher sensitivity and specificity (67\% and 97\%) than T2WI (25\% and 92\%) in the detecting local recurrence. Combining DCE-MRI with DWI further improved tumour detection [43]. DWI also has the potential to evaluate residual disease post-hormone therapy (Fig. 5); this requires further study.

\section{Current limitations of DWI for prostate cancer}

The prostatic base has clinical significance because disease here can extend directly into the seminal vesicles. Evaluation by DWI can be limited by increased cellularity in the normal prostatic base [12]. Kim et al. reported lower sensitivity of DWI for tumour $(66 \%)$ in the prostatic base compared with the TZ (81\%) [43].

Potentially benign conditions that may falsely lower ADC include: (1) various abnormal conditions, such as prostatitis and the compact fibromuscular stroma seen in benign prostatic hyperplasia [10, 44], (2) susceptibility artefact at tissue boundaries [45] and at areas of haemorrhage (Fig. 6), (3) elevated vascular flow associated with tumour [29], as well as (4) organ motion [24]. Conversely, well-differentiated tumours with predominantly glandular composition may yield relatively higher ADC values [17].

Imaging at higher field strengths (e.g. 3T) may increase susceptibility to magnetic field heterogeneity. Nevertheless, a number of recent studies have utilised DWI at 3T with promising results [16, 18, 20, 37, 42, 43] (Fig. 6).

\section{Comparison of DWI imaging with other MR methods}

\section{T2-weighted imaging}

Current literature suggests that DWI, when combined with standard T2-weighted imaging, increases sensitivity and specificity for detecting PC. This may be attributed to increased contrast on DWI images compared with conventional imaging [46]. When combined with T2-weighted imaging, the sensitivity and specificity for PC range from 71 to $89 \%$ and 61 to $91 \%$ respectively, compared with $51-86 \%$ and $60-84 \%$ respectively for T2WI alone [17, 19, 21, 22, 47]. Lim et al. [24] showed that combined T2WI and DWI imaging increased the AUC among readers from $0.66-0.79$ for T2WI alone and $0.74-0.86$ for DWI alone, to $0.76-0.9(p<0.001)$. 

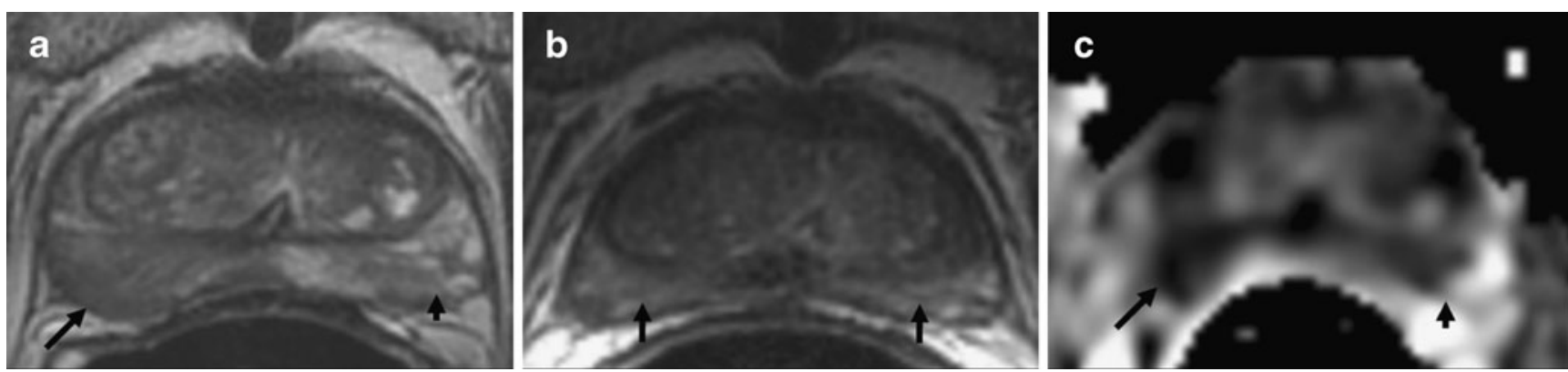

Fig. 5 A 60-year-old man with prostate cancer. MRI was performed using a $1.5 \mathrm{~T}$ scanner. a Before hormone therapy, axial T2WI demonstrates diffuse hypointense signal in the peripheral zone on the right (long arrow) and focal hypointense signal on the left (short arrow). b After hormone therapy, axial T2WI demonstrates diffuse

However, when used in isolation, DWI tends to be less impressive in some studies. DWI alone has a sensitivity of $49-84 \%$ and specificity of $57-93 \%$ compared with a sensitivity of $27-88 \%$ and specificity of $67-91 \%$ for T2WI alone [16, 36, 43, 48]. Furthermore, DWI does not presently achieve the same spatial resolution as T2WI, which is crucial for assessment for extracapsular extension [49]. Hence, at present, DWI should be used as an adjunct to, rather than replace, T2WI. hypointense signal throughout the peripheral zone (arrows), limiting interpretation. The gland has also decreased in size. c After hormone therapy, the ADC map demonstrates low signal in a similar location (long and short arrows) as seen on the pre-treatment axial T2WI

\section{MR spectroscopy}

By determining the chemical composition of the studied tissues, MRS provides metabolic information about prostate tissue. While normal prostate tissue contains a high level of citrate, in PC the citrate level decreases while the choline and creatinine level increases paradoxically. The data are usually presented in the form of graphs in multivoxel analyses. Alternatively, colour coded maps can be super-
Fig. 6 A 68-year-old man with a PSA value of $21 \mathrm{ng} / \mathrm{ml}$. MRI study was obtained using pelvic phased-array coil performed on 3T MRI. a T2-weighted images of prostate gland show focal low signal (arrow) in the right peripheral zone at the level of the mid-gland. This was biopsyproven to represent adenocarcinoma (Gleason's score $4+5$ ). b At $b=50 \mathrm{sec} / \mathrm{mm}^{2}$, the normal peripheral zone retains a high signal, though tumour tissue (arrow) is shown as an area of hyperintensity. c At $b=$ $1,000 \mathrm{sec} / \mathrm{mm}^{2}$, contrast between the tumour (arrow) and the normal peripheral zone is increased. d Corresponding ADC map yielded restricted diffusion in the same region (arrow)
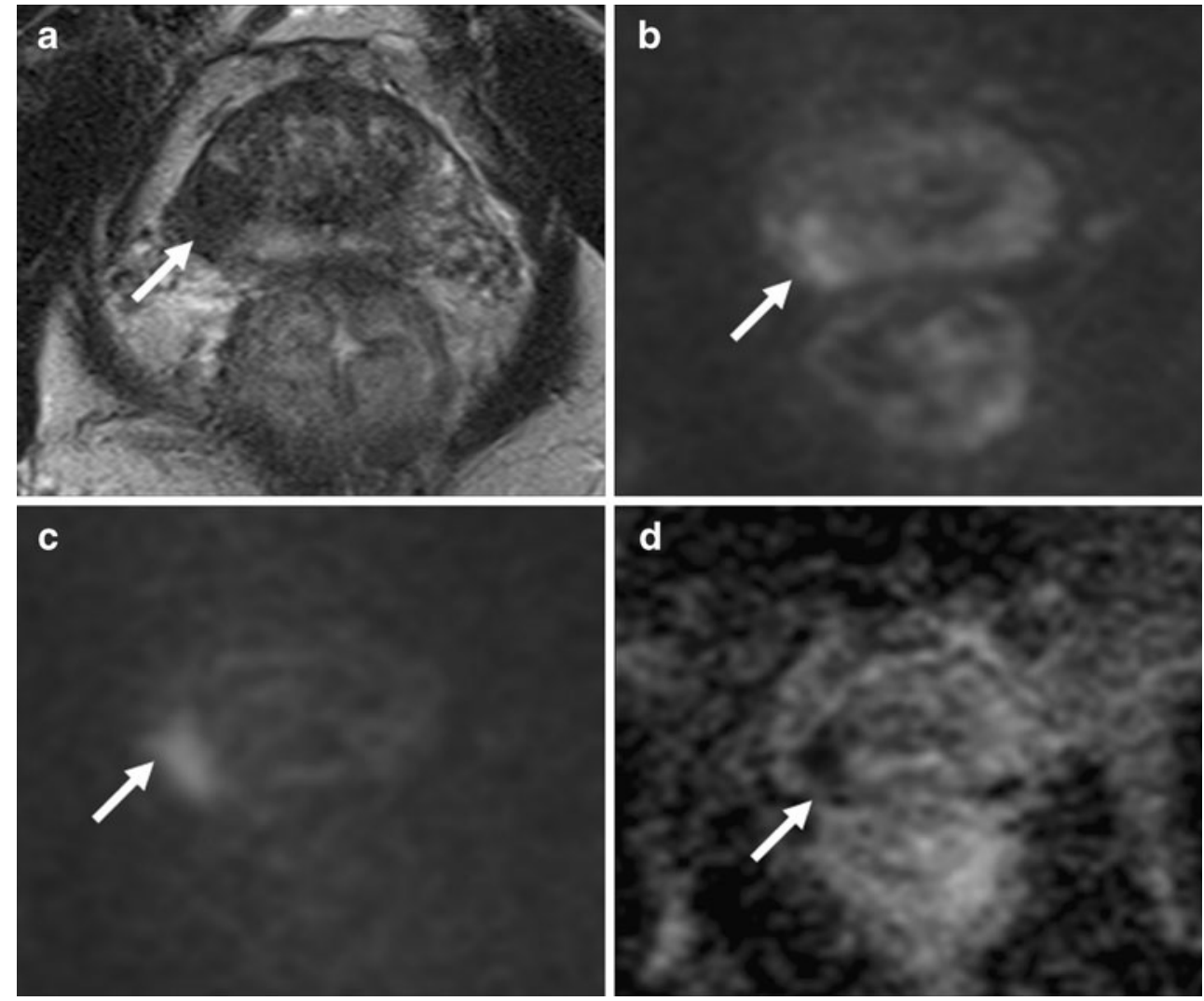
imposed upon T2WI images for anatomic correlation [46]. Compared with DWI, MRS has the disadvantages of longer acquisition time and the need for additional software [46], which lead to increased cost and decreased throughput. Furthermore, signal from periprostatic fat and seminal vesicles can distort spectral waveforms. Lastly, MRS suffers from lack of spatial resolution, estimated to be 15 times poorer than DWI [14]. However, MRS does possess generally acceptable accuracy and diagnostic performance [46].

Results for comparison between DWI and MRS have been mixed. Chen et al. [48] found that DWI $(82 \%)$ and MRS (84\%) had similar sensitivity but DWI had lower specificity (82\%) compared with MRS (98\%). Reinsberg et al. instead showed slightly lower sensitivity and specificity rates of DWI compared with MRS, particularly when the percentage of tumour within the voxel was smaller (see section on voxel size) [50]. Mazaheri et al. found that the AUC for DWI was higher (0.81) than for MRS (0.74), but results were better when both techniques were combined (0.85). Further research directly comparing the two techniques is therefore necessary [14].

\section{Dynamic contrast-enhanced MRI}

DCE-MRI characteristics can be studied either using nonpharmacokinetic (time-intensity curves) and pharmacokinetic methodologies (tracer kinetic modelling). The former method is relatively simpler and can be more easily applied to routine practice, where tumour typically shows early enhancement and washout [51]. The latter method of using tracer kinetic modelling allows for analysis of more sophisticated perfusion parameters, such as mean transit time, blood flow, permeability surface area, and interstitial volume, which have also been shown to be significantly different between PC and normal prostate tissue [52]. To improve ease of use, colour maps of the various complex DCE-MRI parameters can be created and superimposed upon T2WI images for anatomic correlation. Compared with DWI, DCE-MRI has the drawback of intravenous gadolinium administration. Like DWI, it is also limited by motion and biopsy artefacts [53].

The results for DWI compared with DCE-MRI have been mixed. Kim et al. [43] found similar sensitivity and specificity between DWI and DCE-MRI. In another study [42], DWI and T2-weighted imaging combined provided better specificity $(74-78 \%$ versus $63-68 \%)$ but lower sensitivity (63-70\% versus $80-83 \%$ ) compared with DCEMRI. In comparison, Tamada et al. noted better sensitivity by DWI than DCE MRI ( $57 \%$ versus $46 \%$ ), but similar specificity (90\% vs 93\%) [36]. In a more recent study using a $3 \mathrm{~T}$ scanner, by ROC analysis, Kitajima et al. [54] showed that T2WI combined with DCE-MRI improved diagnosis over that T2WI alone, although this difference was not significantly different (AUC 0.87 and 0.84 respectively); however, combined T2WI and DWI as well as combined T2WI, DWI and DCE-MRI (AUC 0.89 and 0.90 respectively) performed statistically significantly better than T2WI alone, but not different from each other (T2WI + DWI versus T2WI + DWI + DCE-MRI). As with MRS, further experience directly comparing DWI and DCE-MRI is needed.

\section{Some technical issues to consider when applying DWI to prostate cancer}

B-value

There is currently no consensus on the optimal b-value to use in prostate DWI $[19,29,46]$. Most studies have used b-values between 0 and $1,000 \mathrm{sec} / \mathrm{mm}^{2}$. In developing DWI into a robust technique for PC imaging, there is a need for standardisation of $b$-values [9].

Higher b-values may provide better characterisation of PC and treatment response in experimental mouse models [55]. Larger b-values are necessary to limit the effects of bulk motion (perfusion) [56]. Qayyam et al. have suggested b-values of more than $1,000 \mathrm{sec} / \mathrm{mm}^{2}$ because of the inherent high T2 signal of the prostate gland [57]. The advantages of using higher b-values include increased diffusion weighting (and diminished T2 shine through) [46], a higher contrast-tonoise ratio, and theoretically, better tumour detection. Disadvantages are increased motion and susceptibility artefacts, as well as decreased signal-to-noise ratios [46, 58].

On the other hand, Kitajima and colleagues found that increasing b-values from $1,000 \mathrm{sec} / \mathrm{mm}^{2}$ to $2,000 \mathrm{sec} / \mathrm{mm}^{2}$ did not confer additional benefit for lesion detection [59]. In neuro-imaging, it has been shown that increasing the b-value from $1,000 \mathrm{sec} / \mathrm{mm}^{2}$ to $3,000 \mathrm{sec} / \mathrm{mm}^{2}$ decreased ADC values by an average of $30 \%$ [60]. Lower signal-tonoise ratios may confound interpretation of different ADC values, as it could be impossible to determine whether the decrease in ADC is due to reduced SNR or true restriction of diffusion. Furthermore, several authors have demonstrated good results using b-values smaller than $1,000 \mathrm{sec} / \mathrm{mm}^{2}$ [29, 39].

$\mathrm{ADC}$ values versus qualitative visual assessment

The ADC is potentially an objective means by which to detect tumours by DWI $[27,39,61,62]$. However, tissue diffusivity depends on both biological (patient's age and body temperature) and technical factors (b-value, location and size of the region of interest) [24, 63]. To date, no definite ADC cut-off for tumours has been established. 
Normalised ADC, the ratio between ADC values of normal and tumour tissue, may potentially overcome variations in patient and technical factors, but has yet to be established [24].

Apparent diffusion coefficient maps are image representations of ADC values and allow for visual comparison between tumour and normal tissue. These have been used with good results in multiple studies [15-18, 20, 22, 24, 47]. Compared with assessing absolute ADC values, this method is less time-consuming, requires less post-processing and may be more practical in the clinical setting. The main limitation is inter-observer variability; therefore, reader experience becomes important [24].

\section{Voxel size}

As DWI reflects tissue composition, the degree of restricted diffusion can be expected to increase with the proportion of tumour within a voxel. Reinsberg et al. [50] noted that DWI with the tumour occupying approximately $30 \%$ of the voxel, sensitivity and specificity were $67 \%$ and $60 \%$, but when tumour occupied approximately $70 \%$ of the voxel, sensitivity and specificity increased to $93 \%$ and $57 \%$ respectively. In the recent study by Langer et al. [64] it was shown that DWI is unable to differentiate between sparse PC (as defined by tumour of less than $40 \%$ within the lesion) and normal PZ tissue. Logically, higher resolution imaging would improve lesion detection, although this needs further validation.

\section{Future directions for DWI in prostate cancer}

\section{Bi-exponential analysis}

Theoretically, mono-exponential analysis, which assumes an inverse linear logarithmic relationship between signal intensity and b-values, could fail to discriminate between the fast and slow components of diffusion. The fast diffusion component (large-scale bulk movement of molecules secondary to capillary flow) is studied at lower b-values. The slow diffusion component (related to the cellularity of tissues) is studied at relatively higher b-values [45]. The bi-exponential method of diffusion analysis theoretically eliminates the effects of perfusion, reflecting tissue diffusion characteristics to a closer degree $[45,65]$. For this method, an extended range of $b$-values $(b>1,000 \mathrm{sec} /$ $\mathrm{mm}^{2}$ ) needs to be acquired. This has been achieved with line scan diffusion imaging, but currently only acquires data at a specific slice location. Currently, its small volume coverage limits this technique for clinical practice [66]. Furthermore, whether bi-exponential analysis confers significant benefit over mono-exponential analysis in practice remains to be shown.
Diffusion tensor imaging

In vivo diffusion tensor imaging (DTI) of the prostate gland is feasible, including that at 3T [67], based on the fact that the motion of water molecules is not truly random, but follows the orientation of the tissue structure (anisotropy) [68]. Gibbs et al. [28] demonstrated increased diffusion in the superior-inferior direction compared with the transaxial plane. PC theoretically disrupts this normal anisotropy. Gibbs and colleagues found that mean fractional anisotropy was significantly increased in tumour (0.24) compared with normal peripheral zone tissue (0.16) [49]. Limitations of DTI include noise variation, long imaging times and specific absorption rate limits $[10,68]$. At present, DTI for PC detection still warrants investigation.

\section{Multiparametric evaluation}

The utility of combining functional MRI techniques with conventional spin echo MRI has been alluded to. Research has been directed towards combining various acquisition parameters (such as water diffusion, chemical environment and vascular properties) into a single value or image to improve tumour localisation [69]. Recently, Langer and colleagues were able to successfully combine quantitative T2WI, DWI and DCE-MRI data through logistic regression to develop an objective model for mapping the tumour within the PZ [62]. Some other authors have shown improvements in accuracy in combined T2WI, DCE-MRI and DWI [47], combined T2WI, DWI and MRS [48], as well as combined T2WI, DCE-MRI and MRS [70], whereas others have not seen as much benefit of two verse three parameter analysis [54]. However, it is currently unclear whether using multiple functional parameters is superior to T2 plus a single parameter such as DWI only, given the increased imaging time and potential expense. This requires further study. Nevertheless, it is anticipated that improvements in scan time acquisition using higher field magnets and dedicated software that can rapidly analyse large amounts of complex data into simpler but easily interpretable information will lead to greater acceptance of multiparametric MRI in routine clinical practice [71].

\section{Conclusion}

Diffusion-weighted imaging is potentially useful in prostate cancer detection and localisation. Early experience suggests that DWI may be useful in localising PC for diagnosis, monitoring, biopsy, treatment planning and staging as well as potentially for response assessment. It may also aid in evaluation of more difficult cases such as disease in the 
transitional zone or when there is haemorrhage. The strengths of this technique are its short imaging time, no need for intravenous contrast material or additional hardware, and relatively simple post-processing requirements. Optimisation and standardisation of the various technical parameters should help render it a robust adjunct to conventional MR imaging of the prostate gland.

\section{References}

1. Horner MJ, Ries LAG, Krapcho M, Neyman N, Aminou R, Howlader N, Altekruse SF, Feuer EJ, Huang L, Mariotto A, Miller BA, Lewis DR, Eisner MP, Stinchcomb DG, Edwards BK (eds) (2009) SEER Cancer Statistics Review, 1975-2006. National Cancer Institute, Bethesda, MD. Available via the SEER web site. http://seer.cancer.gov/csr/1975_2006/, based on November 2008 SEER data submission. Accessed 5 July 2010

2. Mullerad M, Hricak H, Kuroiwa K, Pucar D, Chen HN, Kattan MW, Scardino PT (2005) Comparison of endorectal magnetic resonance imaging, guided prostate biopsy and digital rectal examination in the preoperative anatomical localization of prostate cancer. J Urol 174:2158-2163

3. Wang L, Mullerad M, Chen HN, Eberhardt SC, Kattan MW, Scardino PT, Hricak H (2004) Prostate cancer: incremental value of endorectal MR imaging findings for prediction of extracapsular extension. Radiology 232:133-139

4. Taneja SS (2006) Prostate biopsy: targeting cancer for detection and therapy. Rev Urol 8:173-182

5. Sella T, Schwartz LH, Swindle PW, Onyebuchi CN, Scardino PT, Scher HI, Hricak H (2004) Suspected local recurrence after radical prostatectomy: endorectal coil MR imaging. Radiology 231:379385

6. Mazaheri Y, Shukla-Dave A, Muellner A, Hricak H (2008) MR imaging of the prostate in clinical practice. Magma 21:379-392

7. Engelbrecht MR, Jager GJ, Laheij RJ, Verbeek AL, van Lier HJ, Barentsz JO (2002) Local staging of prostate cancer using magnetic resonance imaging: a meta-analysis. Eur Radiol 12:2294-2302

8. Sonnad SS, Langlotz CP, Schwartz JS (2001) Accuracy of MR imaging for staging prostate cancer: a meta-analysis to examine the effect of technologic change. Acad Radiol 8:149-157

9. Padhani AR, Liu G, Koh DM, Chenevert TL, Thoeny HC, Takahara T, Dzik-Jurasz A, Ross BD, Van Cauteren M, Collins D, Hammoud DA, Rustin GJ, Taouli B, Choyke PL (2009) Diffusion-weighted magnetic resonance imaging as a cancer biomarker: consensus and recommendations. Neoplasia 11:102125

10. Xu J, Humphrey PA, Kibel AS, Snyder AZ, Narra VR, Ackerman JJ, Song SK (2009) Magnetic resonance diffusion characteristics of histologically defined prostate cancer in humans. Magn Reson Med 61:842-850

11. Provenzale JM, Engelter ST, Petrella JR, Smith JS, MacFall JR (1999) Use of MR exponential diffusion-weighted images to eradicate T2 "shine-through" effect. AJR Am J Roentgenol 172:537-539

12. Morgan VA, Kyriazi S, Ashley SE, DeSouza NM (2007) Evaluation of the potential of diffusion-weighted imaging in prostate cancer detection. Acta Radiol 48:695-703

13. Kim CK, Park BK (2008) Update of prostate magnetic resonance imaging at 3 T. J Comput Assist Tomogr 32:163-172

14. Mazaheri Y, Shukla-Dave A, Hricak H, Fine SW, Zhang J, Inurrigarro G, Moskowitz CS, Ishill NM, Reuter VE, Touijer K,
Zakian KL, Koutcher JA (2008) Prostate cancer: identification with combined diffusion-weighted MR imaging and 3D 1H MR spectroscopic imaging-correlation with pathologic findings. Radiology $246: 480-488$

15. Mazaheri Y, Hricak H, Fine SW, Akin O, Shukla-Dave A, Ishill NM, Moskowitz CS, Grater JE, Reuter VE, Zakian KL, Touijer KA, Koutcher JA (2009) Prostate tumor volume measurement with combined T2-weighted imaging and diffusion-weighted MR: correlation with pathologic tumor volume. Radiology 252:449457

16. Miao H, Fukatsu H, Ishigaki $\mathrm{T}$ (2007) Prostate cancer detection with 3-T MRI: comparison of diffusion-weighted and T2-weighted imaging. Eur J Radiol 61:297-302

17. Yoshimitsu K, Kiyoshima K, Irie H, Tajima T, Asayama Y, Hirakawa M, Ishigami K, Naito S, Honda H (2008) Usefulness of apparent diffusion coefficient map in diagnosing prostate carcinoma: correlation with stepwise histopathology. J Magn Reson Imaging 27:132-139

18. Kim CK, Choi D, Park BK, Kwon GY, Lim HK (2008) Diffusionweighted MR imaging for the evaluation of SVI in prostate cancer: initial results. J Magn Reson Imaging 28:963-969

19. Kajihara H, Hayashida Y, Murakami R, Katahira K, Nishimura R, Hamada Y, Kitani K, Kitaoka M, Suzuki Y, Kitajima M, Hirai T, Morishita S, Awai K, Yamashita Y (2009) Usefulness of diffusion-weighted imaging in the localization of prostate cancer. Int J Radiat Oncol Biol Phys 74:399-403

20. Park BK, Lee HM, Kim CK, Choi HY, Park JW (2008) Lesion localization in patients with a previous negative transrectal ultrasound biopsy and persistently elevated prostate specific antigen level using diffusion-weighted imaging at three Tesla before rebiopsy. Invest Radiol 43:789-793

21. Shimofusa R, Fujimoto H, Akamata H, Motoori K, Yamamoto S, Ueda T, Ito H (2005) Diffusion-weighted imaging of prostate cancer. J Comput Assist Tomogr 29:149-153

22. Haider MA, van der Kwast TH, Tanguay J, Evans AJ, Hashmi AT, Lockwood G, Trachtenberg J (2007) Combined T2-weighted and diffusion-weighted MRI for localization of prostate cancer. AJR Am J Roentgenol 189:323-328

23. Ren J, Huan Y, Wang H, Ge Y, Chang Y, Yin H, Sun L (2009) Seminal vesicle invasion in prostate cancer: prediction with combined T2-weighted and diffusion-weighted MR imaging. Eur Radiol 19:2481-2486

24. Lim HK, Kim JK, Kim KA, Cho KS (2009) Prostate cancer: apparent diffusion coefficient map with T2-weighted images for detection-a multireader study. Radiology 250:145-151

25. Kim JH, Kim JK, Park BW, Kim N, Cho KS (2008) Apparent diffusion coefficient: prostate cancer versus noncancerous tissue according to anatomical region. J Magn Reson Imaging 28:11731179

26. Ren J, Huan Y, Li F, Wang H, Ge Y, Chang Y, Yin H, Sun L (2009) Combined T2-weighted and diffusion-weighted MRI for diagnosis of urinary bladder invasion in patients with prostate carcinoma. J Magn Reson Imaging 30:351-356

27. Sato $C$, Naganawa $S$, Nakamura $T$, Kumada $H$, Miura $S$, Takizawa O, Ishigaki T (2005) Differentiation of noncancerous tissue and cancer lesions by apparent diffusion coefficient values in transition and peripheral zones of the prostate. J Magn Reson Imaging 21:258-262

28. Gibbs P, Tozer DJ, Liney GP, Turnbull LW (2001) Comparison of quantitative T2 mapping and diffusion-weighted imaging in the normal and pathologic prostate. Magn Reson Med 46:1054-1058

29. Van As N, Charles-Edwards E, Jackson A, Jhavar S, Reinsberg S, Desouza N, Dearnaley D, Bailey M, Thompson A, Christmas T, Fisher C, Corbishley C, Sohaib S (2008) Correlation of diffusionweighted MRI with whole mount radical prostatectomy specimens. Br J Radiol 81:456-462 
30. Ren J, Huan Y, Wang H, Zhao H, Ge Y, Chang Y, Liu Y (2008) Diffusion-weighted imaging in normal prostate and differential diagnosis of prostate diseases. Abdom Imaging 33:724-728

31. Potter SR, Epstein JI, Partin AW (2000) SVI by prostate cancer: prognostic significance and therapeutic implications. Rev Urol 2:190-195

32. Tempany C, Straus S, Hata N, Haker S (2008) MR-guided prostate interventions. J Magn Reson Imaging 27:356-367

33. Kumar V, Jagannathan NR, Kumar R, Das SC, Jindal L, Thulkar S, Gupta SD, Dwivedi SN, Roell S, Hemal AK, Gupta NP (2006) Correlation between metabolite ratios and ADC values of prostate in men with increased PSA level. Magn Reson Imaging 24:541-548

34. DiBiase SJ, Hosseinzadeh K, Gullapalli RP, Jacobs SC, Naslund MJ, Sklar GN, Alexander RB, Yu C (2002) Magnetic resonance spectroscopic imaging-guided brachytherapy for localized prostate cancer. Int J Radiat Oncol Biol Phys 52:429-438

35. Pouliot J, Kim Y, Lessard E, Hsu IC, Vigneron DB, KurhanewiTZ J (2004) Inverse planning for HDR prostate brachytherapy used to boost dominant intraprostatic lesions defined by magnetic resonance spectroscopy imaging. Int $\mathrm{J}$ Radiat Oncol Biol Phys 59:1196-1207

36. Tamada T, Sone T, Jo Y, Yamamoto A, Yamashita T, Egashira N, Imai S, Fukunaga M (2008) Prostate cancer: relationships between postbiopsy hemorrhage and tumor detectability at MR diagnosis. Radiology 248:531-539

37. Zelhof B, Pickles M, Liney G, Gibbs P, Rodrigues G, Kraus S, Turnbull L (2009) Correlation of diffusion-weighted magnetic resonance data with cellularity in prostate cancer. BJU Int 103:883-888

38. Gibbs P, Liney GP, Pickles MD, Zelhof B, Rodrigues G, Turnbull LW (2009) Correlation of ADC and T2 measurements with cell density in prostate cancer at 3.0 Tesla. Invest Radiol 44:572-576

39. deSouza NM, Riches SF, Vanas NJ, Morgan VA, Ashley SA, Fisher C, Payne GS, Parker C (2008) Diffusion-weighted magnetic resonance imaging: a potential non-invasive marker of tumour aggressiveness in localized prostate cancer. Clin Radiol 63:774-782

40. Rouvière $\mathrm{O}$, Mège-Lechevallier $\mathrm{F}$, Chapelon JY, Gelet $\mathrm{A}$, Bouvier R, Boutitie F, Lyonnet D (2006) Evaluation of color Doppler in guiding prostate biopsy after HIFU ablation. Eur Urol 50:490-497

41. Chen J, Daniel BL, Diederich CJ, Bouley DM, van den Bosch MA, Kinsey AM, Sommer G, Pauly KB (2008) Monitoring prostate thermal therapy with diffusion-weighted MRI. Magn Reson Med 59:1365-1372

42. Kim CK, Park BK, Lee HM, Kim SS, Kim E (2008) MRI techniques for prediction of local tumor progression after highintensity focused ultrasonic ablation of prostate cancer. AJR Am J Roentgenol 190:1180-1186

43. Kim CK, Park BK, Park W, Kim SS (2010) Prostate MR imaging at $3 \mathrm{~T}$ using a phased-arrayed coil in predicting locally recurrent prostate cancer after radiation therapy: preliminary experience. Abdom Imaging 35:246-252

44. Charles-Edwards EM, deSouza NM (2006) Diffusion-weighted magnetic resonance imaging and its application to cancer. Cancer Imaging 6:135-143

45. Riches SF, Hawtin K, Charles-Edwards EM, de Souza NM (2009) Diffusion-weighted imaging of the prostate and rectal wall: comparison of biexponential and monoexponential modelled diffusion and associated perfusion coefficients. NMR Biomed 22:318-325

46. Choi YJ, Kim JK, Kim N, Kim KW, Choi EK, Cho KS (2007) Functional MR imaging of prostate cancer. Radiographics 27:63-77

47. Tanimoto A, Nakashima J, Kohno H, Shinmoto H, Kuribayashi S (2007) Prostate cancer screening: the clinical value of diffusionweighted imaging and dynamic MR imaging in combination with T2-weighted imaging. J Magn Reson Imaging 25:146-152
48. Chen M, Dang HD, Wang JY, Zhou C, Li SY, Wang WC, Zhao WF, Yang ZH, Zhong CY, Li GZ (2008) Prostate cancer detection: comparison of T2-weighted imaging, diffusion-weighted imaging, proton magnetic resonance spectroscopic imaging, and the three techniques combined. Acta Radiol 49:602-610

49. Gibbs P, Pickles MD, Turnbull LW (2006) Diffusion imaging of the prostate at 3.0 Tesla. Invest Radiol 41:185-188

50. Reinsberg SA, Payne GS, Riches SF, Ashley S, Brewster JM, Morgan VA, deSouza NM (2007) Combined use of diffusionweighted MRI and 1H MR spectroscopy to increase accuracy in prostate cancer detection. AJR Am J Roentgenol 188:9198

51. Engelbrecht MR, Huisman HJ, Laheij RJ, Jager GJ, van Leenders GJ, Hulsbergen-Van De Kaa CA, de la Rosette JJ, Blickman JG, Barentsz JO (2003) Discrimination of prostate cancer from normal peripheral zone and central gland tissue by using dynamic contrast-enhanced MR imaging. Radiology 229:248-254

52. Buckley DL, Roberts C, Parker GJ, Logue JP, Hutchinson CE (2004) Prostate cancer: evaluation of vascular characteristics with dynamic contrast-enhanced T1-weighted MR imaging-initial experience. Radiology 233:709-715

53. White S, Hricak H, Forstner R et al (1995) Prostate cancer: effect of postbiopsy hemorrhage on interpretation of MR images. Radiology 195:385-390

54. Kitajima K, Kaji Y, Fukabori Y, Yoshida K, Suganuma N, Sugimura K (2010) Prostate cancer detection with 3 T MRI: comparison of diffusion-weighted imaging and dynamic contrastenhanced MRI in combination with T2-weighted imaging. J Magn Reson Imaging 31:625-631

55. Roth Y, Tichler T, Kostenich G, Ruiz-Cabello J, Maier SE, Cohen JS, Orenstein A, Mardor Y (2004) High-b-value diffusion-weighted MR imaging for pretreatment prediction and early monitoring of tumor response to therapy in mice. Radiology 232:685-692

56. Issa B (2002) In vivo measurement of the apparent diffusion coefficient in normal and malignant prostatic tissues using echoplanar imaging. J Magn Reson Imaging 16:196-200

57. Qayyum A (2009) Diffusion-weighted Imaging in the Abdomen and Pelvis: Concepts and Applications. Radiographics 1797-1810

58. Naganawa S, Kawai H, Fukatsu H, Sakurai Y, Aoki I, Miura S, Mimura T, Kanazawa H, Ishigaki T (2005) Diffusion-weighted imaging of the liver: technical challenges and prospects for the future. Magn Reson Med Sci 4:175-186

59. Kitajima K, Kaji Y, Kuroda K, Sugimura K (2008) High b-value diffusion-weighted imaging in normal and malignant $\mathrm{PZ}$ tissue of the prostate: effect of signal-to-noise ratio. Magn Reson Med Sci 7:93-99

60. DeLano MC, Cooper TG, Siebert JE, Potchen MJ, Kuppusamy K (2000) High-b-value diffusion-weighted MR imaging of adult brain: image contrast and apparent diffusion coefficient map features. AJNR Am J Neuroradiol 21:1830-1836

61. Hosseinzadeh K, Schwarz SD (2004) Endorectal diffusion-weighted imaging in prostate cancer to differentiate malignant and benign peripheral zone tissue. J Magn Reson Imaging 20:654-661

62. Langer DL, van der Kwast TH, Evans AJ, Trachtenberg J, Wilson BC, Haidr MA (2009) Prostate cancer detection with multiparametric MRI: logistic regression analysis of quantitative T2, diffusion-weighted imaging, and dynamic contrast-enhanced MRI. J Magn Reson Imaging 30:327-334

63. Bilgili Y, Unal B (2004) Effect of region of interest on interobserver variance in apparent diffusion coefficient measures. AJNR Am J Neuroradiol 25:108-111

64. Langer DL, van der Kwast TH, Evans AJ, Sun L, Yaffe MJ, Trachtenberg J, Haider MA (2008) Intermixed normal tissue within prostate cancer: effect on MR imaging measurements of apparent diffusion coefficient and T2-sparse versus dense cancers. Radiology 249:900-908 
65. Mulkern RV, Barnes AS, Haker SJ, Hung YP, Rybicki FJ, Maier SE, Tempany CM (2006) Biexponential characterization of prostate tissue water diffusion decay curves over an extended b-factor range. Magn Reson Imaging 24:563-568

66. Shinmoto H, Oshio K, Tanimoto A, Higuchi N, Okuda S, Kuribayashi S, Mulkern RV (2009) Biexponential apparent diffusion coefficients in prostate cancer. Magn Reson Imaging 27:355-359

67. Sinha S, Sinha U (2004) In vivo diffusion tensor imaging of the human prostate. Magn Reson Med 52:530-537

68. Gürses B, Kabakci N, Kovanlikaya A, Firat Z, Bayram A, Uluğ AM, Kovanlikaya I (2008) Diffusion tensor imaging of the normal prostate at 3 Tesla. Eur Radiol 18:716-721
69. Chan I, Wells W 3rd, Mulkern RV, Haker S, Zhang J, Zou KH, Maier SE, Tempany CM (2003) Detection of prostate cancer by integration of line-scan diffusion, T2-mapping and T2-weighted magnetic resonance imaging; a multichannel statistical classifier. Med Phys 30:2390-2398

70. Turkbey B, Pinto PA, Mani H, Bernardo M, Pang Y, McKinney YL, Khurana K, Ravizzini GC, Albert PS, Merino MJ, Choyke PL (2010) Prostate cancer: value of multiparametric MR imaging at 3 $\mathrm{T}$ for detection-histopathologic correlation. Radiology 255:89-99

71. Kurhanewicz J, Vigneron D, Carroll P, Coakley F (2008) Multiparametric magnetic resonance imaging in prostate cancer: present and future. Curr Opin Urol 18:71-77 